\title{
Cellular automata model for emergent properties of pressure flow in single nephron compliance tubule
}

\author{
Siva Manohar Reddy Kesu ${ }^{1}$, Hariharan Ramasangu ${ }^{2}$ \\ ${ }^{1}$ Department of Electronics and Communication, Faculty of Engineering and Technology, \\ M. S. Ramaiah University of Applied Sciences, Bengaluru, India \\ ${ }^{2}$ Research Division, Relecura Inc, Bengaluru, India
}

\begin{abstract}
Article Info
Article history:

Received Sep 8, 2021

Revised Dec 13, 2021

Accepted Jan 11, 2022

Keywords:

Autoregulation

Cellular automata

Compliance tubule

Emergent properties

ABSTRACT

Numerical analysis plays a vital role in the computational modeling of a nephron. The solutions from numerical methods exhibit regular, period doubling, and irregular oscillations as global behavior. A single nephron compliance tubular model with transport mechanism and autoregulatory mechanism has been developed using the cellular automata framework in this paper. Global emergent behavior of the biological system has been captured using cellular automata framework. An ultradiscretization technique is used to convert the governing partial differential equations of a single nephron compliance tubular model to cellular automata local rules. The global emergent behaviors from the local cellular automata rules have been compared with the reported experimental analysis. The cellular automata framework in biological modeling is an emerging perspective to model global functional behaviors.
\end{abstract}

Nephron

Ultradiscretization

This is an open access article under the CC BY-SA license.

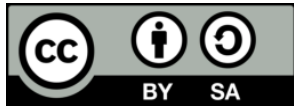

\section{Corresponding Author:}

Siva Manohar Reddy Kesu

Department of Electronics and Communication, Faculty of Engineering and Technology

M. S. Ramaiah University of Applied Sciences

Bengaluru, India

Email: smreddyeee@cvsr.ac.in

\section{INTRODUCTION}

Kidneys maintain the autoregulation of the blood pressure which may vary due to fluctuations caused by the blood vessels and heartbeats [1]. The blood flow in the kidney drives the glomerular filtration and is then autoregulated by several mechanisms to stabilize the transport mechanism [2]. The numerical methods and the large computing power available are used to simulate the transport mechanism of compliance nephrons. Models are refined to increase the accuracy at the tubular level by incorporating more individual transporters. Mathematical models of single nephron compliance tubular function have been developed in detail at the microscale level by incorporating many segments of the nephron such as thin ascending limb, thick ascending limb and descending limb [3]. This helps in capturing the detailed functionality of a nephron at structural and hemodynamic levels based on the transportation of the water and electrolytes. Macroscopic mathematical models of nephron with water, sodium chloride and urea transport have been tested with the experimental conditions and revealed the process of urine concentrating mechanism [4], [5].

Layton [6] have developed a model to represent tubular chloride concentration because the solute concentration at the macula densa (MD) is the primary signal that initiates the Tubuloglomerular feedback mechanism (TGF) autoregulatory response [6]. Single nephron TGF compliance tubular models are primarily based on the models of Holstein-Rathlou and Marsh [7]. The main focus of the models is to determine the factors that contribute to sustained oscillatory behavior that has been observed experimentally. Sustained 
oscillations occur in the compliance tubule model when the resistance of the afferent arteriole is included in the governing differential equations.

Moss et al. [8] has developed a new approach for the modeling of nephron system using onedimensional network automata. The Moss model incorporates the discretized structural and behavior of the nephron for fluid flow, solute transport and TGF. The discretized model has captured the self-sustained oscillations, period doubling and irregular oscillations with various TGF gain and has been compared with the A. T. Layton's continuous nephron models [6].

Experimental analysis has been extensively conducted by Leyssac and Holstein on Sprague-Dawley (SD) rats, Winstar Kyoto (WKY) rats and spontaneously hypertensive (SH) rats [9], [10]. They have identified that the oscillation in the nephron tubule occurs due to autoregulatory mechanisms such as myogenic and TGF mechanisms. Regular stable oscillatory behavior has been observed in the SpragueDawley rats under the experimental conditions by giving various dosages of loop diuretics. The loop diuretics regulate the autoregulatory mechanism from low concentration to high concentration to get regular oscillatory property, period doubling and irregular oscillatory behavior in the SD, WKY and SH rats [9], [10].

Cellular automata (CA) models are useful in modeling the emergent behavior of an evolving system. The CA models have been applied to model various phenomena [11], [12]. The key step in creating a CA model is to arrive at appropriate local space-time evolution rules. In this paper, a nephron model has been proposed with myogenic and TGF autoregulatory system that explicitly illustrates the functionality of the nephron tubule with compliant tubular walls using cellular automata. We have modeled pressure-driven flow within a compliant tubule using cellular automata based on the compliant-TGF model developed by Layton and Edwards [13].

The CA model captures the emergent behavior during the evolution in terms of the template of 256 elementary rules. The properties from the rules developed for the governing partial differential equation (PDE) have to be compared with 256 rules and analyse the global emergent behavior [14]. The properties exhibited by the cellular automata model are not obvious for the analysis of emergent behavior [15]. The continuous governing equations are discretized and the CA model is then constructed using ultradiscretization (UD) [16]. The UD procedure preserves the integrability and the characteristics of integrability [11]. The UD equations with the algebraic setting have been applied in the max-plus algebra, min-plus algebra and minimax algebra [3], [17].

In this paper, we have developed a systematic procedure to construct a single nephron compliance tubular model from differential equations to CA rules with appropriate assumptions. Only sodium chloride as solute concentration is considered and the other electrolytes such as potassium and bicarbonates, which have a low impact on the autoregulatory mechanism, are assumed to be absent. The solutions developed by the rules from the cellular automata model have been analysed and compared with the original experimental findings. The developed single nephron compliance tubular model in this paper is able to capture the regular, period doubling and irregular pulse propagation properties, which have been observed in the experimental analysis.

\section{CELLULAR AUTOMATA BASED SINGLE NEPHRON COMPLIANCE TUBULAR MODEL}

We have described the governing equations related to the single nephron model in this section. The schematic diagram of a single nephron model, shown in Figure 1, comprises of glomerulus, pre-ascending limb, thick ascending limb (TAL) and autoregulatory mechanism. Blood enters glomerulus through the afferent arteriole and is filtered before passing to the efferent arteriole. The filtrates flow through the preascending limb, and then go to the TAL. After TAL, the solutes go to the collecting duct through MD. The information related to the solute concentration is fed back to the afferent arteriole from MD. This feedback process is called the TGF mechanism. The active transport dynamics in TAL play a major role in TGF regulation of the solute concentration. The afferent arteriole contracts and expands due to myogenic and TGF mechanisms [18], [19].

Layton and Edwards [13] has given the modified governing equations for the glomerulus and nephron tubule. The governing equations are formed using nephron tubular fluid pressure $P(x, t)$; tubular fluid volumetric flow rate $Q(x, t)$; nephron tubular radius $R(x, t)$, and fluid solute concentration $C(x, t)$ as dependent variables [8], [13], [20], [21]. Boundary conditions for tubular fluid pressure are needed at the two ends of the TAL. The inflow fluid pressure in TAL is determined by TGF response. The tubular fluid pressure is assumed at the end of the TAL based on the pressure range observed in previous experiments. The TAL extends from $x=0$ at loop-bend to $x=L$ at the early distal tubule end (MD) [21]. The single nephron tubule extends in space from the loop-bend to the collecting duct end, where tubular fluid pressure in rats has shown to be $13 \mathrm{mmHg}$ based on interstitium measurements. The total length of the nephron tubule is represented as $L_{0}$ which equals $4 L$. The nephron inflow pressure $P(0, t)=P_{0}(t)$ and the outflow pressure $P\left(L_{0}, t\right)=P_{1}(t)$ are considered as boundary conditions. This advection-diffusion nephron tubule in (1) for the pressure $\mathrm{P}$ was advanced in time using numerical analysis [13]. 


$$
\begin{aligned}
& \frac{\partial P(x, t)}{\partial t}=\frac{R^{3}(x, t)}{16 \mu \frac{d R}{d P}} \frac{\partial^{2} P(x, t)}{\partial x^{2}}+\frac{R^{2}(x, t)}{4 \mu \frac{d R}{d P}} \frac{\partial R(x, t)}{\partial x} \frac{\partial P(x, t)}{\partial x} \\
& \frac{\partial}{\partial t}\left(\pi R^{2}(x, t) C(x, t)=-\frac{\partial}{\partial x} Q(x, t) C(x, t)-2 \pi R_{S S}(x)\left(\frac{V_{\max }(x) C(x, t)}{K_{M}+C(x, t)}+\kappa\left(C(x, t)-C_{e}(x)\right)\right)\right.
\end{aligned}
$$

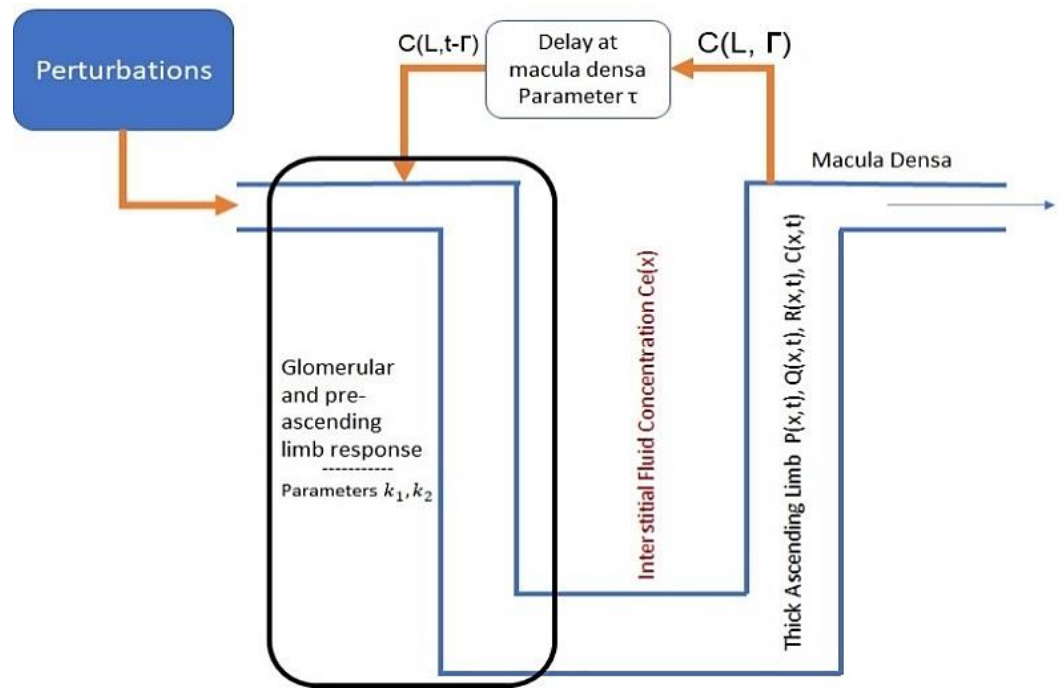

Figure 1. Block diagram of a compliance nephron tubule with modeling parameters

The modeling equation [21], As shown in (2), represents the solute concentration in TAL. The time independent interstitial solute concentration is represented as $C_{e}(x)$. The active transport mechanism is incorporated through maximum active transport rate $V_{\text {max }}(x)$ (Michaelis-Menten-like kinetics) and Michaelis constant $K_{M}$. The transepithelial solute diffusion is denoted by backleak permeability $\kappa$. The solute transport mechanism in TAL is independent of $R(x, t)$ and is directly proportional to the steady state TAL radius $R_{S S}(x)$. The boundary condition $C(0, t)=C_{0}$ means that the fluid entering into the TAL has a constant solute concentration.

$$
R(x, t)=\alpha\left(P(x, t)-P_{e}\right)+\beta(x)
$$

$P_{e}$ is the interstitial pressure along the TAL, $\alpha$ is the degree of tubular compliance of the nephron tubule, and $\beta(x)$ is the unpressurized TAL radius.

$$
P_{0}(t)=P_{0}\left(1+K_{1} \tanh \left(K_{2}\left(C_{o p}-C(L, t-\tau)\right)\right)\right.
$$

As shown in (3) and (4) show the myogenic and TGF mechanism for the complaint single nephron tubule respectively. $C(L, t-\tau)=C_{o p}$ is taken as initial condition when $t=0 . K_{1}$ is the half range of pressure variation $P_{0}(t)$ and $K_{2}$ denotes the TGF sensitivity. $C_{o p}$ is steady state TAL solute concentration at MD. $C(L, t-\tau)$ is solute concentration along MD, where $\tau$ represents TGF delay [21]. The model parameters and their values along with the auxiliary equations, are as in Ryu [21].

\subsection{Ultradiscretization of compliance nephron tubular model}

In this section, the cellular automata model for pressure and solute concentration governing equations has been developed. The partial differential equations are first transformed by ultradiscretization. The binary transformation is then applied to the ultradiscretized variables.

The tubular pressure in (1) of the nephron tubular model has been simplified and shown in (5) with the consideration of $k_{1}=\frac{1}{16 \mu \frac{d R}{d P}}$ and $k_{2}=\frac{1}{4 \mu \frac{d R}{d P}}$.

$$
\frac{\partial P(x, t)}{\partial t}=k_{1} R^{3}(x, t) \frac{\partial^{2} P(x, t)}{\partial x^{2}}+k_{2} R^{2}(x, t) \frac{\partial R(x, t)}{\partial x} \frac{\partial P(x, t)}{\partial x}
$$


In (5) has a negativity problem that cannot be ultradiscretized with normal tropical discretization. This issue has been resolved by applying Cole-Hopf transformation to (7) where c is a constant.

$$
\begin{aligned}
& P=\frac{f_{x}}{f} \\
& P(j \Delta x,(n+1) \Delta t)=c \frac{f((j+1) \Delta x, n \Delta t}{f(j \Delta x, n \Delta t)}
\end{aligned}
$$

Let us denote $\delta=k_{1} \frac{\Delta t}{\Delta x^{2}}$ and $m=\frac{1-2 \delta}{c \delta}$.

$$
\begin{aligned}
& \Omega= 1+\frac{1-2 \delta}{c \delta} P(j \Delta x, n \Delta t) R^{3}(j \Delta x, n \Delta t) \\
&+k_{2} \frac{1}{c^{2}} P((j+1) \Delta x, n \Delta t) R((j+1) \Delta x, n \Delta t) R^{2}(j \Delta x, n \Delta t) \\
& \Lambda= 1+\frac{1-2 \delta}{c \delta} P((j-1) \Delta x, n \Delta t) R^{3}((j-1) \Delta x, n \Delta t) \\
&+k_{2} \frac{1}{c^{2}} P(j \Delta x, n \Delta t) R(j \Delta x, n \Delta t) R^{2}((j-1) \Delta x, n \Delta t) \\
& P(j \Delta x,(n+1) \Delta t)=P((j-1) \Delta x, n \Delta t) \frac{\Omega}{\Lambda}
\end{aligned}
$$

Let us use $\frac{1-2 \delta}{c \delta}=e^{\frac{-M}{\epsilon}}, c^{2}=e^{\frac{L_{1}}{\epsilon}}, P(j \Delta x, n \Delta t)=e^{\frac{P_{1}(j \Delta x, n \Delta t)}{\epsilon}}$ and $R(j \Delta x, n \Delta t)=e^{\frac{R_{1}(j \Delta x, n \Delta t)}{\epsilon}}$. These equations have been substituted in (10) and in the limit when $\epsilon \rightarrow+0$. We get (11) after the application of Min-Max algebra and assuming $L_{1}-K_{2}=N$.

$$
\begin{aligned}
& P_{1}(j \Delta x,(n+1) \Delta t)=P_{1}((j-1) \Delta x, n \Delta t)+2 R_{1}(j \Delta x, n \Delta t)+\max \left[2 R_{1}(j \Delta x, n \Delta t),\right. \\
& \quad P_{1}(j \Delta x, n \Delta t)+3 R_{1}(j \Delta x, n \Delta t)-M, P_{1}((j+1) \Delta x, n \Delta t)+ \\
& \left.\quad R_{1}((j+1) \Delta x, n \Delta t)-N\right]-2 R_{1}((j-1) \Delta x, n \Delta t)-\max \left[2 R_{1}((j-1) \Delta x, n \Delta t),\right. \\
& \left.\quad P_{1}((j-1) \Delta x, n \Delta t)+3 R_{1}((j-1) \Delta x, n \Delta t)-M, P_{1}(j \Delta x, n \Delta t)+R_{1}(j \Delta x, n \Delta t)-N\right]
\end{aligned}
$$

\subsection{Cellular automata rules for single nephron tubular model}

The CA rule has been developed for (10) with the consideration of the nephron tubule as compliance tubular $R(x, t)=P(x, t)$. The cellular automata model parameters are calculated from the corresponding experimental findings [6]. The possible case for the CA rule has been prepared based on the model parameters and the case is $N \ll M$, where $\mathrm{N}$ and $\mathrm{M}$ are CA parameters for pressure oscillations. When we consider nephron tubule as compliance tubule and assume $R(j \Delta x, n \Delta t)=P(j \Delta x, n \Delta t)$ where $N \ll M$ and substitute in (11), we get (12).

$$
\begin{gathered}
P_{1}(j \Delta x,(n+1) \Delta t)=P_{1}((j-1) \Delta x, n \Delta t)+2 P_{1}(j \Delta x, n \Delta t)+\max \left[2 P_{1}(j \Delta x, n \Delta t),\right. \\
\left.4 P_{1}(j \Delta x, n \Delta t)-M, 2 P_{1}((j+1) \Delta x, n \Delta t)-N\right]-2 P_{1}((j-1) \Delta x, n \Delta t)- \\
\max \left[2 P_{1}((j-1) \Delta x, n \Delta t), 4 P_{1}((j-1) \Delta x, n \Delta t)-M, 2 P_{1}(j \Delta x, n \Delta t)-N\right]
\end{gathered}
$$

The eight possibilities have been substituted in the ultradiscretized pressure (12) and the outputs have been depicted in Table 1. Table 1 gives the CA Rule 206 for the ultradiscretized as shown in (12) with the assumption nephron tubule as compliance tubule where $R(j \Delta x, n \Delta t)=P(j \Delta x, n \Delta t)$.

Table 1. Cellular automata approach to calculate pressure $P_{1}(j \Delta x,(n+1) \Delta t)$ variations for compliance tubule

\begin{tabular}{ccccc}
\hline S1 No & $P_{1}((j-1) \Delta x, n \Delta t)$ & $P_{1}(j \Delta x, n \Delta t)$ & $\left.P_{1}(j+1) \Delta x, n \Delta t\right)$ & $P_{1}(j \Delta x,(n+1) \Delta t)$ \\
\hline 1 & $N$ & $N$ & $N$ & $N$ \\
2 & $N$ & $N$ & $M$ & $M$ \\
3 & $N$ & $M$ & $N$ & $M$ \\
4 & $N$ & $M$ & $M$ & $M$ \\
5 & $M$ & $N$ & $N$ & $N$ \\
6 & $M$ & $N$ & $M$ & $M$ \\
7 & $M$ & $M$ & $N$ & $M$ \\
8 & $M$ & $M$ & $M$ & \\
\hline
\end{tabular}


The output of each row from Table 1 has to be noted from the most significant bit (MSB) to the least significant bit (LSB) in order to obtain CA rule. The order from MSB to LSB is $M M N N M M M N$ and converted into binary form as 11001110 . The binary form value is converted into the decimal value as 206. The value 206 obtained is the rule number from the elementary cellular automata rule framework developed by Wolfram [22].

\section{RESULTS AND DISCUSSION}

Single nephron model with transport mechanism and autoregulation has been modeled using cellular automata. The physiological behavior of the single nephron model has been analysed for the compliance tubular model. The behavior of the model has been explicitly studied with and without autoregulation.

Leyssac and Holstein have performed the experiments on the SD, SH and WKY rats [9], [10]. Pressure variations have been observed in the rats after reducing the solute active transport mechanism using loop diuretics [10]. The data collected by the investigators strongly support that the pressure oscillation developed in the afferent arteriole is due to the TGF mechanism initiated by the solute concentration at the MD. These observations were analysed by micro-puncturing at the proximal convolution tubule.

In the cellular automata, the evolution takes place in a spatio-temporal grid. The origin cell is the left topmost cell. The spatial increment is along the X-axis (towards right) and the temporal increment is along the $\mathrm{Y}$-axis (towards down). The dynamic state of cells are represented by white (0) and black (1) where black represents the cell value is above the threshold and white represents the cell value is below the threshold. Initialization in CA means assigning black cells in the first two rows. The first row initialization is for nofeedback case and the first two-rows initialization is for TGF case. The evolutionary patterns arrived from the CA rules have been categorized and compared with the Wolfram CA class framework based on Table 2.

Table 2. CA evolution schemes for various initializations with TGF $(\checkmark)$ and without feedback (X)

\begin{tabular}{ccc}
\hline Initialization Scheme & First row & Second row \\
\hline IC1 & Left top & X \\
IC2 & Left top & 2-cell spacing \\
IC3 & 2-cell spacing & X diagonally right $(\checkmark)$ \\
IC4 & 2-cell spacing & 1-cell right shift $(\checkmark)$ \\
IC5 & Only middle & 1-cell left shift $(\checkmark)$ \\
IC6 & Only middle & X \\
IC7 & Random & X \\
IC8 & Random & Random $(\checkmark)$ \\
IC9 & 4-cell symmetry spacing & X \\
IC10 & 4-cell symmetry spacing from center4-cell symmetry spacing from center $(\checkmark)$ \\
IC11 & X \\
IC12 & Alternate cell spacing & 1-cell right and 1-cell left shift $(\checkmark)$ \\
IC13 & Alternate cell spacing & 1-cell right and 1-cell left shift $(\checkmark)$ \\
\hline
\end{tabular}

The physiological interpretations of CA rule 206 will be discussed for the compliance nephron tubular model. CA rules are derived for pressure variations with various initialization schemes depicted in Table 3, selected out of many possibilities, for the study of the evolution of patterns from the CA rule 206. The initialization means there is a pressure perturbation as black in the initial section of the tubule at $t=0$ for TGF inhibited case. The pressure perturbation has been initialized with black cells at $t=0$ in the first layer and also perturbation as black has been initiated in the second layer below the previous section at $t=1$ for TGF activated case. The evolved patterns in Figures 2, 3, and 4 have been analysed and are shown in Table 3 that exhibit the emergent global properties of the physiological behavior of the nephron tubule.

Table 3. Physiological interpretations obtained from various patterns in figures for nephron compliance model

\begin{tabular}{|c|c|c|c|}
\hline Figures & Emergent Behavior [Proposed CA model] & $\begin{array}{l}\text { Wolfram } \\
\text { Class }\end{array}$ & $\begin{array}{l}\text { Conditions under which the emergent behavior has been observed } \\
\text { experimentally }\end{array}$ \\
\hline \multicolumn{3}{|c|}{ Figure 2(a)Pulse is not propagating and spatially constant Class 2} & Not available \\
\hline Figure 2(b) & Pulse inhibition & Class 1 & Normal ATF fluid in WKY rats [9]. \\
\hline Figure 2(c) & Period doubling behavior & Class 2 & Medium TGF activation in SD rat [9], [10]. \\
\hline Figure 3(a) & Pulses are constant and are not sustained & Class 2 & Not available. \\
\hline Figure $3(\mathrm{~b})$ & Unstable pulsating behavior & Class 4 & $10-20$ mins after injection of $7.5 \times 10^{-4}$ moles of furosemide [9]. \\
\hline Figure $3(\mathrm{c})$ & Regular pulsating behavior & Class 2 & $5-8$ mins after stopping TGF mechanism in SHR rats [9]. \\
\hline Figure 4(a) & Stable oscillatory behavior & Class 2 & Within 3 mins of ATF injection in WKY rats [9]. \\
\hline Figure $4(\mathrm{~b})$ & Irregular and unstable pulses & Class 2 & $3-5$ mins after injection of $3.0 \times 10^{-4}$ moles of furosemide [9]. \\
\hline Figure $4(\mathrm{c})$ & Propagated irregularly & Class 3 & $5-10$ mins after injection of $7.5 \times 10^{-4}$ moles of furosemide [9]. \\
\hline
\end{tabular}




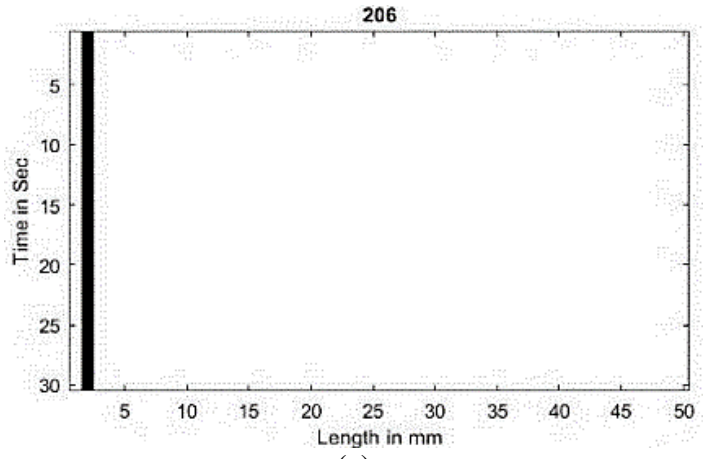

(a)

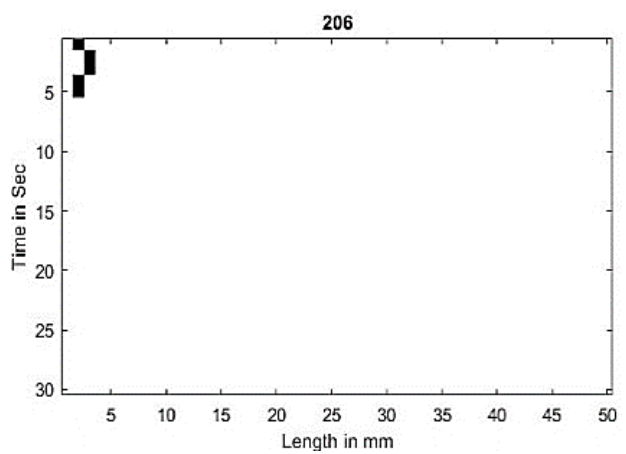

(b)

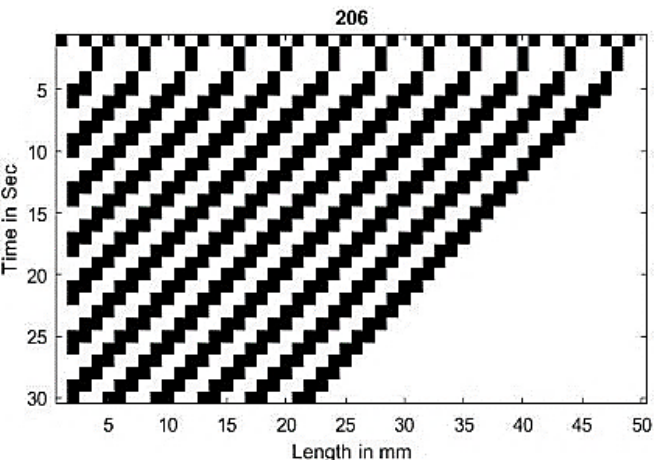

(c)

Figure 2. Pressure perturbation pattern in nephron tubule using CA analysis with (a) initialization of IC1 scheme without feedback, (b) initialization of IC2 scheme with TGF mechanism, and (c) TGF is activated when IC13 scheme is initialized

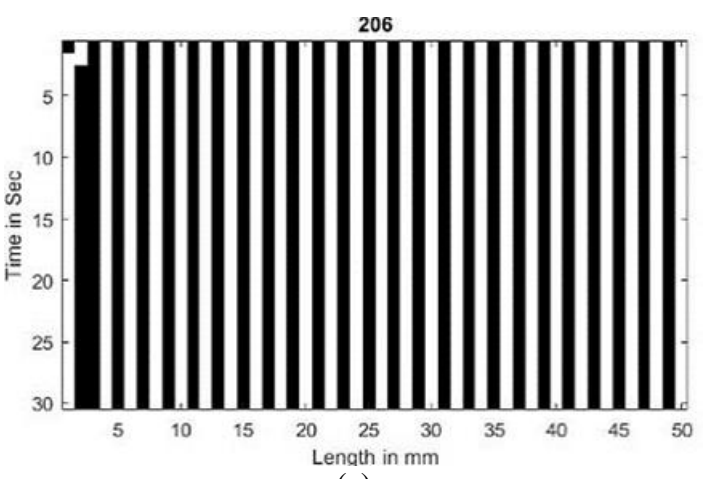

(a)

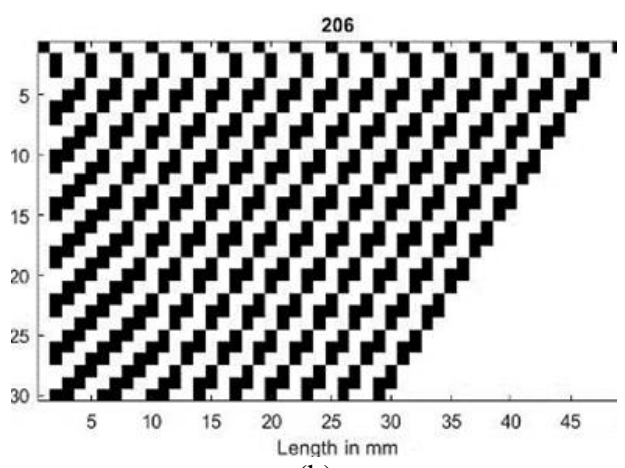

(b)

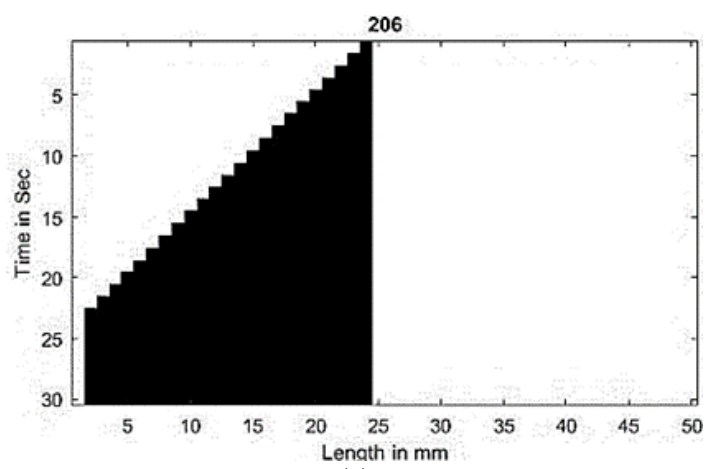

(c)

Figure 3. Pressure perturbation pattern in nephron tubule using CA analysis with (a) TGF is not activated when IC12 scheme is initialized, (b) IC5 scheme when TGF is activated case, and (c) IC6 scheme when no-TGF case 
The study of the pressure variations in the nephron tubule under various concentrations of loop diuretics exhibits self-sustained oscillation, period-doubling oscillation, and irregular oscillation in the experimental findings. Moreover, these oscillations have been observed in different evolutionary patterns, shown in figures obtained from the cellular automata rule are depicted in Table 3. In summary, pressure perturbation behavior in the nephron tubule has shown that the oscillations are of different regimes such as regular, stable, period doubling, and irregular. This global physiological behavior has been observed from the CA analysis with a simple set of local rules with various initialization schemes shown in Table 3 . The regular pressure oscillatory behavior has been observed in the normotensive rats whereas hypertensive rats exhibit chaotic and non-period oscillatory properties [23].

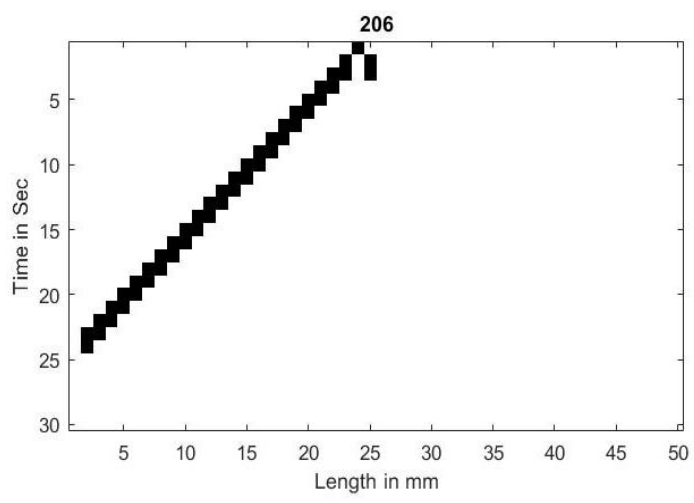

(a)

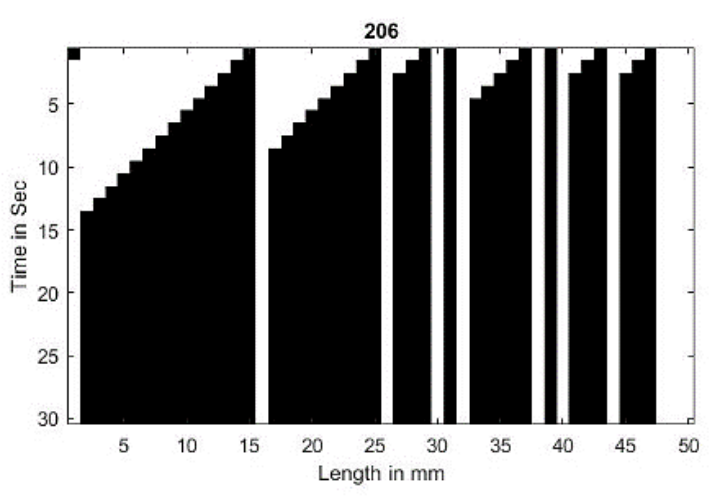

(b)

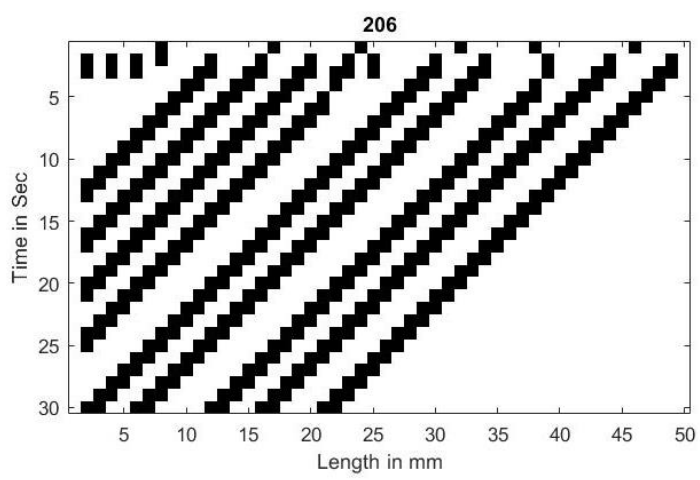

(c)

Figure 4. Pressure perturbation pattern in nephron tubule using CA analysis with (a) IC7 scheme is initialized with TGF case, (b) IC8 scheme initialization without feedback, and (c) TGF is activated when IC9 scheme is initialized

The whole kidney model has to be developed for better physiological and pathological behavior using cellular automata. Such model can then be compared with the Robert Moss and Gale Thomas whole kidney model for better understanding [8], [24]. In this work, an attempt has been made to view the nephron modeling from the point of view of cellular automata. Moreover, the cellular automata approach offers a way to incorporate the learning mechanism at the local level and allow the system to evolve for studying the global behavior [12], [25]. Physiological process modeling with learning mechanism as a fundamental unit suggests an integrated approach for living systems [26]. The learning algorithm, as modeling tool compared to task-based ones, is yet to take off in a big way. The proposed CA approach will be extended to explore the possibilities.

\section{CONCLUSION}

The single nephron compliance tubular model has been developed by transforming the governing partial differential equations to cellular automata rules. Ultradiscretization techniques are used to transform the governing equations to the corresponding cellular automata rules. The proposed cellular automata based 
nephron compliance tubular model exhibits emergent behavior that has been experimentally observed. The nephron model with compliance tubule is found to be associated with rule number 206 in the CA scheme. The evolved behavior from the cellular automata nephron model has been compared with the experimental findings. The pressure perturbations in the nephron tubule, under different assumptions, exhibit regular to irregular pulsating behavior due to the two auto-regulatory mechanisms - TGF and myogenic in compliance tubular model. The modeling approach of cellular automata can be extended to multinephron modeling to understand the whole kidney physiology.

\section{REFERENCES}

[1] V. A. J. Douglas Eaton and Pooler John, Renal Functions, Anatomy, and Basic Processes, 6th ed. New York: Vander's renal physiology, 2009.

[2] S. Chen and A. Zaikin, "Modelling Complex Phenomena in Physiology," in Quantitative Physiology, Singapore: Springer Singapore, 2020, pp. 189-237.

[3] A. Edwards, "Modeling transport in the kidney: investigating function and dysfunction," American Journal of Physiology-Renal Physiology, vol. 298, no. 3, pp. F475-F484, Mar. 2010, doi: 10.1152/ajprenal.00501.2009.

[4] A. T. Layton, H. E. Layton, W. H. Dantzler, and T. L. Pannabecker, "The Mammalian Urine Concentrating Mechanism: Hypotheses and Uncertainties," Physiology, vol. 24, no. 4, pp. 250-256, Aug. 2009, doi: 10.1152/physiol.00013.2009.

[5] D. J. Marsh, D. D. Postnov, O. V. Sosnovtseva, and N.-H. Holstein-Rathlou, "The nephron-arterial network and its interactions," American Journal of Physiology-Renal Physiology, vol. 316, no. 5, pp. F769-F784, May 2019, doi: 10.1152/ajprenal.00484.2018.

[6] A. T. Layton, "Modeling Transport and Flow Regulatory Mechanisms of the Kidney," ISRN Biomathematics, vol. 2012, pp. 1-18, Aug. 2012, doi: 10.5402/2012/170594.

[7] S. J. Graybill, "Modelling nephron dynamics and tubuloglomerular feedback," University of Canterbury, Christchurch, New Zealand, 2010.

[8] R. Moss, E. Kazmierczak, M. Kirley, and P. Harris, “A computational model for emergent dynamics in the kidney,” Philosophical Transactions of the Royal Society A: Mathematical, Physical and Engineering Sciences, vol. 367, no. 1896, pp. 2125-2140, Jun. 2009, doi: 10.1098/rsta.2008.0313.

[9] N.-H. Holstein-Rathlou and P. P. Leyssac, "TGF-mediated oscillations in the proximal intratubular pressure: differences between spontaneously hypertensive rats and Wistar-Kyoto rats," Acta Physiologica Scandinavica, vol. 126, no. 3, pp. 333-339, Mar. 1986, doi: 10.1111/j.1748-1716.1986.tb07824.x.

[10] P. P. Leyssac and N. H. Holstein-Rathlou, "Effects of various transport inhibitors on oscillating TGF pressure responses in the rat," Pflügers Archiv European Journal of Physiology, vol. 407, no. 3, pp. 285-291, Sep. 1986, doi: 10.1007/BF00585304.

[11] M. Murata, "Tropical discretization: ultradiscrete Fisher-KPP equation and ultradiscrete Allen-Cahn equation," Journal of Difference Equations and Applications, vol. 19, no. 6, pp. 1008-1021, Jun. 2013, doi: 10.1080/10236198.2012.705834.

[12] N. V M and L. Jeganathan, "A symmetry based anomaly detection in brain using cellular automata for computer aided diagnosis," Indonesian Journal of Electrical Engineering and Computer Science, vol. 14, no. 1, p. 471, Dec. 2018, doi: 10.11591/ijeecs.v14.i1.pp471-477.

[13] A. T. Layton and A. Edwards, Mathematical Modeling in Renal Physiology. Berlin, Heidelberg: Springer Berlin Heidelberg, 2014.

[14] S. Wolfram, A new kind of science, vol. 5. IL: Wolfram media, 2002.

[15] K. Matsuya and M. Murata, "Spatial pattern of discrete and ultradiscrete Gray-Scott model," Discrete \& Continuous Dynamical Systems - B, vol. 20, no. 1, pp. 173-187, 2015, doi: 10.3934/dcdsb.2015.20.173.

[16] S. Ohmori and Y. Yamazaki, "Cellular Automata for Spatiotemporal Pattern Formation from Reaction-Diffusion Partial Differential Equations," Journal of the Physical Society of Japan, vol. 85, no. 1, p. 014003, Jan. 2016, doi: 10.7566/JPSJ.85.014003.

[17] S. Jin, L. Xu, C. Xu, and D. Ma, "Lane width-based cellular automata model for mixed bicycle traffic flow," Computer-Aided Civil and Infrastructure Engineering, vol. 34, no. 8, pp. 696-712, Aug. 2019, doi: 10.1111/mice.12445.

[18] J. Schnermann and J. P. Briggs, "Tubuloglomerular feedback: mechanistic insights from gene-manipulated mice," Kidney International, vol. 74, no. 4, pp. 418-426, Aug. 2008, doi: 10.1038/ki.2008.145.

[19] S. M. R. Kesu and H. Ramasangu, "Improved Nephron Model for Pressure-Diuresis and Pressure-Natriuresis," in 2016 IEEE 16th International Conference on Bioinformatics and Bioengineering (BIBE), Oct. 2016, pp. 192-197, doi: 10.1109/BIBE.2016.59.

[20] R. Moss and S. R. Thomas, "Hormonal regulation of salt and water excretion: a mathematical model of whole kidney function and pressure natriuresis," American Journal of Physiology-Renal Physiology, vol. 306, no. 2, pp. F224-F248, Jan. 2014, doi: 10.1152/ajprenal.00089.2013.

[21] H. Ryu, "Feedback-Mediated Dynamics in the Kidney: Mathematical Modeling and Stochastic Analysis," Duke University, Durham, NC, 2014.

[22] S. Wolfram, Cellular automata and complexity. CRC Press, 2018

[23] K. H. Chon, R. Raghavan, Y.-M. Chen, D. J. Marsh, and K.-P. Yip, "Interactions of TGF-dependent and myogenic oscillations in tubular pressure," American Journal of Physiology-Renal Physiology, vol. 288, no. 2, pp. F298-F307, Feb. 2005, doi: 10.1152/ajprenal.00164.2004.

[24] T. Gale, "Simulation of whole mammalian kidneys using complex networks," University of Melbourne, 2016.

[25] E. AlKaldy, A. H. Majeed, M. S. Zainal, and D. M. Nor, "Optimum multiplexer design in quantum-dot cellular automata," Indonesian Journal of Electrical Engineering and Computer Science, vol. 17, no. 1, p. 148, Jan. 2020, doi: 10.11591/ijeecs.v17.i1.pp148-155.

[26] A. Aljuboori, L. A. Tawfeeq, and K. A. Al-Karawi, "Pushing towards ehealth for iraqi hypertensives: an integrated class association rules into SECI model," Indonesian Journal of Electrical Engineering and Computer Science, vol. 22, no. 1, p. 522, Apr. 2021, doi: 10.11591/ijeecs.v22.i1.pp522-533. 


\section{BIOGRAPHIES OF AUTHORS}

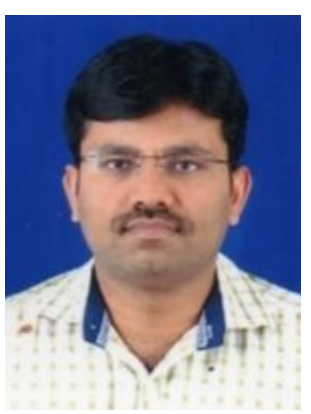

Siva Manohar Reddy Kesu (D) SI SC P is a doctoral student at M S Ramaiah University of Applied Sciences, Bangalore, India. He obtained M. Sc [Engg] from M S Ramaiah School of Advanced Studies (Coventry University), Bangalore, India in 2010. His research interest includes kidney modelling, mathematical physiology, cellular learning automata and deep learning. He can be contacted at email: smreddyeee@cvsr.ac.in.

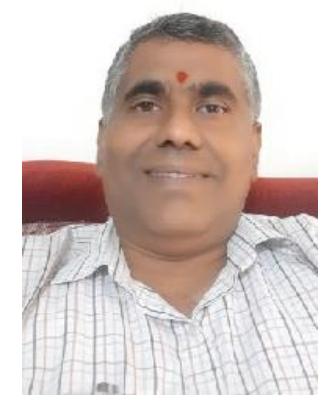

Hariharan Ramasangu (iD Id SC P received his Ph.D (2007) from the Indian Institute of Science. He has 25+ years of experience in academic and research work. He was the head of the Department of Electronics and Communication Engineering and head of the Center for Machine Learning and Computational Intelligence at Ramaiah University of Applied Sciences, Bangalore, India. Before that, he worked at the National Institute of Securities Market and National Aerospace Laboratories, India. He has over 40 publications and 10 patents. Currently, he is the director of research at Relecura. He can be contacted at email: rharihar@ ieee.org. 